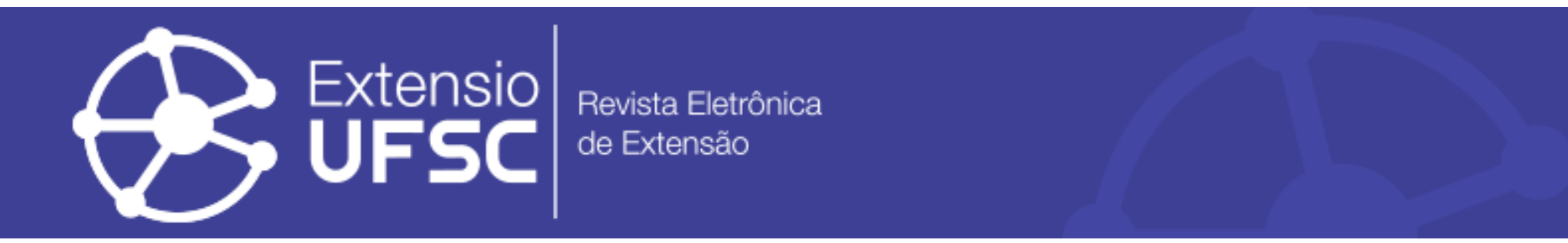

\title{
CONCEITOS E PRÁTICAS EM SAÚDE: A ENFERMAGEM COMEMORANDO O DIA INTERNACIONAL DA SAÚDE
}

\author{
Jeane Barros de Souza \\ Universidade Federal da Fronteira Sul \\ jeanebarros18@gmail.com \\ Ângela Urio \\ Universidade Federal da Fronteira Sul \\ ange.urio@hotmail.com \\ Maraisa Manorov \\ Universidade Federal da Fronteira Sul \\ mara_manorov@hotmail.com
}

Daniela Savi Geremia Universidade Federal da Fronteira Sul daniela.geremia@uffs.edu.br

Adriana Hillesheim Universidade Federal da Fronteira Sul adriana.hillesheim@uffs.edu.br

Valéria Silvana Faganello Madureira Universidade Federal da Fronteira Sul valeria.madureira@uffs.edu.br

\begin{abstract}
Resumo
Tem-se por objetivo compartilhar a experiência do planejamento e execução de atividades em comemoração ao Dia Mundial da Saúde na universidade. As atividades foram organizadas por estudantes e docentes de graduação em enfermagem, de uma universidade federal do oeste catarinense, em abril de 2018. Foram organizadas três rodas de conversa na entrada do hall principal da universidade, oportunizando a participação de aproximadamente 40 pessoas em cada turno. Sobre o significado de saúde, os participantes consideraram o completo bem-estar biopsicossocial, o direito a alimentação, lazer, moradia, trabalho, bem como acesso ao sistema de saúde. Alguns participantes consideraram que a saúde mental pode ser prejudicada devido à pressão, ao estresse e às exigências da vida acadêmica. Torna-se imprescindível a continuidade de atividades como essa que oportunizem discussões sobre diversos temas, tanto na universidade como em outros espaços comunitários.

Palavras-chave: Saúde. Universidade. Enfermagem. Determinantes de Saúde.
\end{abstract}

\section{CONCEPTS AND PRACTICES IN HEALTH: NURSING CELEBRATING INTERNATIONAL HEALTH DAY}

\begin{abstract}
The objective is to share the experience of planning and executing activities in celebration of World Health Day at the university. The activities were organized by students and undergraduate nursing professors from a federal university in the west of Santa Catarina in April 2018. Three talk wheels were organized at the entrance to the main hall of the university, with the participation of approximately 40 people in each shift. On the meaning of health, participants considered the complete biopsychosocial well-being, the right to food, leisure, housing, work, as well as access to the health system. Some participants felt that mental health can be hampered by pressure, stress and the demands of academic life. It is imperative to continue activities such as this that allow discussions on various topics, both in the university and in other community spaces.
\end{abstract}

Keywords: Health. University. Nursing. Determinants of Health.

\section{CONCEPTOS Y PRÁCTICAS EN SALUD: LA ENFERMATE COMEMORANDO EL DÍA INTERNACIONAL DE LA SALUD}

\begin{abstract}
Resumen
Se tiene por objetivo compartir la experiencia de la planificación y ejecución de actividades en conmemoración del Día Mundial de la Salud en la universidad. Las actividades fueron organizadas por estudiantes y docentes de graduación en enfermería, de una universidad federal del oeste catarinense, en abril de 2018. Se organizaron tres ruedas de conversación en la entrada del hall principal de la universidad, dando oportunidad a la participación de aproximadamente 40 personas en cada turno. En cuanto al significado de salud, los participantes consideraron el completo bienestar biopsicosocial, el derecho a la alimentación, el ocio, la vivienda, el trabajo, así como el acceso al sistema de salud. Algunos participantes consideraron que la salud mental puede ser perjudicial debido a la presión, el estrés y las exigencias de la vida académica. Se hace imprescindible la continuidad de actividades como esa que oportunicen discusiones sobre diversos temas, tanto en la universidad y en otros espacios comunitarios.
\end{abstract}

Palavras clave: Salud. Universidad. Enfermería. Determinantes de Salud. 


\section{INTRODUÇÃO}

O conceito de saúde é multidimensional, reflete a conjuntura social, econômica, política, cultural e não representa a mesma coisa para todas as pessoas (SCLIAR, 2007). A Organização Mundial da Saúde (OMS, 1946) define saúde não apenas como a ausência de doença, mas como o perfeito bem-estar físico, mental e social. A amplitude desse conceito gerou críticas de natureza técnica, política ou libertária, as quais apontavam que o conceito permitia abusos por parte do Estado, pois lhe possibilitava intervir na vida dos cidadãos sob o pretexto de promover a saúde. Em decorrência da primeira objeção, surgiu o conceito de Christopher Boorse (1977) definindo saúde como a ausência de doença. No entanto, a partir desse conceito, a classificação dos seres humanos como saudáveis ou doentes passou a ser unicamente uma questão objetiva relacionada à eficiência das funções biológicas. Resposta a isso foi dada pela declaração final da Conferência Internacional de Assistência Primária à Saúde, realizada na cidade Alma-Ata, em 1978, promovida pela OMS (SCLIAR, 2007). Essa Conferência enfatizou as desigualdades na situação de saúde entre países desenvolvidos e subdesenvolvidos, destacando a responsabilidade governamental na provisão da saúde e a importância da participação de pessoas e comunidades no planejamento e implementação dos cuidados (WHO, 1986).

Em 1986, a Primeira Conferência Internacional sobre Promoção da Saúde, em Ottawa, baseou-se em conceito ampliado de saúde que envolvia condicionantes e determinantes do processo saúde-doença e avançava para além da percepção de saúde como ausência de doença. A Carta de Ottawa, resultado dessa Conferência, reforça a relação indissolúvel entre população e meio no processo saúde-doença, com base em uma abordagem socioecológica da saúde. Saúde é entendida como um princípio orientador e estimulante da efetiva contemplação de questões relacionadas aos agravos que as injustiças sociais e os problemas ambientais produzem na saúde, assim como a criação de ambientes favoráveis (BEZERRA; SORPRESO, 2016). Em 1988, a definição de saúde registrada no artigo 196 da Constituição Federal do Brasil afirma que saúde é direito de todos e dever do Estado, a ser garantido por políticas sociais e econômicas que visem à redução do risco de doença e de outros agravos e ao acesso universal e igualitário às ações e serviços para a promoção, proteção e recuperação (BRASIL, 1988). Tal conceito norteou a criação do Sistema Único de Saúde (SUS).

É importante salientar que o conceito de saúde da OMS foi divulgado na carta de princípios, no dia 7 de abril de 1948. Desde então, o Dia Mundial da Saúde passou a ser comemorado neste dia, traduzindo o reconhecimento do direito à saúde e da obrigação do Estado em promovê-la e protegê-la. Esse dia visa conscientizar as pessoas sobre a importância da 
prevenção e da promoção da saúde para uma melhor qualidade de vida (QV). Em 2018, o tema escolhido pela OMS foi "Saúde para todas e todos, em todos os lugares” (VICTOR, 2018).

Em vista disso, estudantes do curso de graduação em Enfermagem e docentes realizaram ações no espaço universitário em comemoração ao Dia Mundial da Saúde. A partir das vivências exitosas nasceu esse artigo para compartilhar a experiência de planejamento e execução das atividades na universidade, com o intuito de promover discussões e reflexões sobre o conceito de saúde, seus determinantes e práticas adotadas pelos participantes para obter saúde na vida pessoal, familiar e comunitária.

\section{MATERIAIS E MÉTODOS}

Trata-se de um relato de experiência de ações desenvolvidas no mês de abril de 2018, em comemoração ao Dia Mundial da Saúde, em uma universidade federal do oeste catarinense. As atividades foram organizadas e realizadas por estudantes da $5^{\text {a }}$ (quinta) e da $9^{\text {a }}$ (nona) fases do curso de enfermagem e docentes integrantes de um grupo de pesquisa da instituição. No primeiro momento, para o planejamento das atividades, realizou-se uma reunião com os membros do grupo para definir as ações a serem realizadas, bem como o local, a disponibilidade de horários dos envolvidos e os meios de divulgação. Após várias sugestões, optou-se pela realização de rodas de conversa, considerando que essa metodologia permite a partilha de experiências e o desenvolvimento de reflexões sobre as vivências de cada um, em um processo mediado pela interação com os pares, por meio de diálogos internos e no silêncio observador e reflexivo (MOURA; LIMA, 2014).

Então, uma agenda foi programada para que as rodas de conversa ocorressem nos três períodos (matutino, vespertino e noturno), oportunizando a participação do público universitário e envolvimento de todos os cursos da instituição. Para facilitar a visibilidade da ação, as rodas de conversa ocorreram na entrada do hall principal da universidade, com decoração colorida e criativa para chamar a atenção do público (estudantes, docentes e técnicos). Assim, no início do dia, o ambiente foi decorado com murais, cadeiras dispostas em círculo e cartazes, o que despertou a atenção das pessoas que passavam pelo local. Também foi realizado um convite, via aplicativo de mensagens por celular para vários grupos a fim de divulgar a ação no campus universitário. Com vistas a instigar a participação do público, definiram-se duas questões a serem abordadas em uma enquete com o público universitário, a saber: ‘O que é saúde?’ e ‘Como você classifica sua saúde?’ 
As rodas de conversa aconteceram às 10 horas, às 15 horas e às 19 horas, com rodízio das equipes. No início de cada turno, os estudantes responsáveis pelas atividades acompanhados por um docente realizaram uma enquete com as pessoas que circulavam na universidade, convidando-as também a participar das rodas de conversa e a responder as duas questões supracitadas. Após, as respostas obtidas em cada turno foram escritas em tarjetas e anexadas nos murais para serem posteriormente discutidas nas rodas de conversa.

Em cada turno, participaram das atividades cerca de quarenta (40) pessoas. Para iniciar, as organizadoras se apresentaram e falaram da proposta da roda de conversa. Após a apresentação das perguntas e das respostas obtidas, debateu-se sobre essas questões, relacionando-as ao conceito ampliado de saúde, ressaltando a promoção da saúde e a prevenção de doenças, bem como abrindo espaço para discussão do tema. Para finalizar, realizaram-se alongamento físico e abraços comunitários.

\section{RESULTADOS E ANÁLISES}

\section{Reflexões sobre o conceito de saúde}

Em resposta à questão sobre o significado de saúde, a maioria dos participantes da atividade afirmou não considerar somente a falta de doença, mas o completo bem-estar biopsicossocial, conforme definição estabelecida pela OMS. Segundo Araújo e Xavier (2014), o conceito de saúde designado pela OMS cumpriu o objetivo de propor e gerar consenso entre as nações sobre o que deveria ou não ser considerado doença. Permitiu ainda que as dicotomias entre corpo e mente, biológico e social, saúde e doença fossem superadas ao valorizar a importância das esferas social e psicológica no estado pleno de saúde. Contudo, esse é um conceito muito amplo e subjetivo, pois é impossível identificar e delimitar o que dever ser aceito como bem-estar ou mal-estar psicossocial, sendo que as concepções variam de acordo com as percepções e significados que cada pessoa atribui à vida. Por sua vez, tais percepções e significados estão diretamente relacionados às condições materiais e objetivas de vida de cada um, especialmente em um país de dimensões continentais, multicultural e com grandes desigualdades como o Brasil.

Entretanto, outros integrantes das rodas de conversa referiram que ter saúde é ter direito a alimentação, lazer, moradia, trabalho, bem como acesso ao sistema de saúde, concepção que vai ao encontro do conceito ampliado de saúde definido pela $8^{a}$ Conferência Nacional de Saúde (CNS). De acordo com o relatório final dessa conferência, saúde é resultado "das condições de 
alimentação, habitação, educação, renda, meio ambiente, trabalho, transporte, lazer, emprego, liberdade, acesso e posse da terra e acesso a serviços de saúde" (BRASIL, 1986, p. 4). Esse conceito reconhece a relação direta entre situação de saúde e condições de vida e trabalho dos indivíduos e de grupos da população ao abordar os determinantes sociais de saúde (BUSS; PELLEGRINI FILHO, 2007). A definição enfatiza também que saúde não é conceito abstrato, pois se define no contexto de uma sociedade, em um dado momento de seu desenvolvimento e é conquistada nas lutas cotidianas da população (BRASIL, 1986).

Esse conceito ampliado de saúde evidencia como determinantes e condicionantes de saúde elementos da vida cotidiana nem sempre acessíveis a todos. Para efetivá-lo é preciso que a população se aproprie dele, o que demanda conscientização, educação para a cidadania e controle social, para que o direito básico à saúde e seus determinantes sejam reivindicados e alcançados (ALMEIDA, 2013). Entretanto, além das instâncias coletivas de participação, reivindicação e controle social há que haver o esforço e a vontade individual de manter e ou melhorar sua própria saúde e QV. Trata-se de um duplo movimento que se dá, ao mesmo tempo, em duas direções: do coletivo, da macroestrutura para o viver cotidiano de cada um e vice-versa. Daí a importância de discutir 'saúde' com a comunidade para possibilitar e estimular a reflexão crítica sobre o processo saúde-doença e sobre a atual situação do sistema de saúde.

Embora a maioria dos participantes não tenha relacionado 'ter saúde' apenas à ausência de doenças, percebeu-se que durante as discussões, muitos fatores relacionados à garantia de direitos e à produção de saúde ainda são assuntos pouco conhecidos, o que reforça a necessidade de reflexão. Fica evidente a necessidade de estabelecer mais canais de interação com a população que permitam divulgar a concepção ampliada de saúde e problematizá-la, possibilitando, além da compreensão do funcionamento e estrutura do SUS, a apropriação do modelo proposto.

\section{Afinal, como está sua saúde?}

Ao abordar a qualidade de saúde do público universitário, as respostas foram variadas, pois alguns consideraram ter boa saúde, enquanto outros consideraram razoável/regular e outros, ruim/desequilibrada. Os que afirmaram ter boa qualidade de saúde basearam suas percepções no fato de ter uma alimentação saudável e praticar atividade física. Tais elementos são relacionados ao estilo de vida, estão implicados na determinação do processo saúde e doença.

A qualidade de vida é um conceito amplo, que compreende a saúde física, o estado psicológico, o nível de independência e as relações sociais do indivíduo e suas interfaces com o meio onde está inserido, abrangendo sua percepção quanto à posição que ocupa no contexto da 
cultura e dos sistemas de valores que está envolvido, bem como os seus objetivos, expectativas, preocupações e padrões (WHO, 1994).

Portanto, existe uma relação comumente estabelecida entre saúde e QV posto que ficam subentendidos elementos descritos no conceito de saúde da $8^{a}$ CNS, já citado. Tais elementos são explicitados nos domínios propostos pela OMS para avaliação da QV em diferentes culturas, quais sejam: domínio físico, domínio psicológico, nível de independência, relações sociais, meioambiente e aspectos espirituais/religião/crenças pessoais (FLECK, 2000). Houve também quem apontasse ter boa saúde por não apresentar doenças associadas. No entanto, a OMS afirma que saúde é um estado de completo bem-estar físico, mental e social e não apenas a ausência de doença ou enfermidade (WHO, 1986). Essa forma de perceber sua própria saúde dá relevo aos aspectos biológicos relacionados ao bom funcionamento do corpo.

As avaliações da própria saúde como razoável/regular e como ruim/desequilibrada feitas pelos participantes que evidenciaram a falta de atividade física, bem como a alimentação não saudável, o que gera aumento de peso. Destaca-se que, para os universitários, a saída da casa dos pais, a mudança de local de residência, as exigências da vida acadêmica e as novas redes sociais que passam a integrar tendem a modificar seus hábitos de vida e favorecem a adoção de condutas consideradas nocivas à saúde, dentre as quais a diminuição da atividade física e o baixo consumo de alimentos saudáveis (FONTES; VIANNA, 2009). A esse respeito, estudo desenvolvido por Deliens et al. (2015) concluiu que os estudantes universitários participantes não entendiam o significado de comportamento sedentário, o que dificulta a valorização de suas implicações na saúde. Estudo de Lansini et al. (2017) evidenciou que grande número de universitários do Rio Grande do Sul são sedentários, resultados similares aos encontrados em estudos com estudantes de outras regiões brasileiras. Já no agreste pernambucano, Ramos et al. (2019) identificaram níveis moderados de atividade física em estudantes universitários da área da saúde, especialmente no sexo masculino.

As avaliações de sua própria saúde feitas pelos participantes evidenciam a relação entre estilo de vida e saúde, elemento central na abordagem do Ministério da Saúde (MS) para prevenção e cuidado a pessoas com doenças crônicas. Não é recente a discussão sobre a QV dos estudantes, pois, já em 1989, Nahas afirmava que os universitários tendiam a não se preocupar com a saúde, ressaltando que isso poderia ser visto como um problema, já que hábitos da vida adulta são, em grande parte, estabelecidos durante a juventude (NAHAS, 1989).

Os participantes das rodas de conversa também discutiram a respeito da saúde mental, que é prejudicada devido à pressão, ao estresse e às exigências da vida acadêmica. O ingresso no ensino superior gera mudanças no cotidiano do estudante, proporcionando novas experiências 
associadas a novos e distintos sentimentos, o que influencia a sua percepção em relação a sua QV e bem-estar (SILVA; HELENO, 2012).

Revisão integrativa sobre sofrimento psíquico em estudantes universitários e fatores associados concluiu que características da vida acadêmica (semestre do curso, percepção negativa do ambiente, expectativa ruim quanto ao futuro, avaliações, muitas horas de estudo) aliadas a outros aspectos da saúde dos universitários (tabagismo, dieta inadequada, sedentarismo) associaram-se à ocorrência de transtornos mentais comuns. Por outro lado, o apoio social foi decisivo na menor ocorrência de sofrimento psíquico. Assim, aspectos da vida acadêmica aliados a fatores ligados ao estilo de vida podem comprometer a saúde física e mental dos estudantes, bem como sua aprendizagem (GRANER; CERQUEIRA, 2019). Padovani et al. (2014, p.8) corroboram essa posição ao concluírem que os sinais e sintomas de estresse, burnout, ansiedade e depressão comumente encontrados dentre os estudantes universitários, revelam sofrimento psicológico que, por sua vez, "pode ter implicações consideráveis no processo de aprendizagem e formação do futuro profissional".

\section{Possibilidades de aprendizagem}

A atividade envolveu estudantes de enfermagem no planejamento e no desenvolvimento. Tal envolvimento foi intencional justamente porque atividades dessa natureza oportunizam a aprendizagem e o desenvolvimento de habilidades e competências. A participação nessa atividade, com o nível de envolvimento e com a responsabilidade requisitada, proporcionou o aprendizado do tema em questão, pois exigiu preparo de todos para colocar-se em diálogo com outras pessoas e outros saberes, com condições de aprofundar a discussão e a reflexão sobre a temática. Além disso, inseriu os estudantes no contexto da gestão, posto que demandou elementos necessários a ela, tais como trabalho em equipe, planejamento, organização, liderança e tomada de decisões.

Envolver os estudantes na elaboração e execução de toda a atividade proporcionou diferentes formas de aprendizado, pois foram vários encontros, discussões, mudanças de planos, o que expôs os universitários a diferentes situações e demandou estratégias de tomada de decisões. Ações desta natureza promovem a apropriação do saber, o trabalho em equipe, a gestão da atividade proposta, bem como envolve o estudante em um contexto comunitário diferente daquele evidenciado em sala de aula, cenários de prática ou em pesquisas.

A condução das rodas de conversa foi responsabilidade dos estudantes com acompanhamento de um docente, permitindo que seus conhecimentos fossem colocados à prova 
propiciando auto avaliação, bem como a oportunidade de atuação em educação em saúde para públicos fora da sala de aula e dos cenários práticos. Dada a relevância do tema, a comunidade acadêmica foi surpreendida ao ser abordada por colegas estudantes de enfermagem que conduziram as discussões com muita propriedade.

A realização da atividade no espaço universitário trouxe visibilidade ao conceito de saúde e ao curso de enfermagem, proporcionando integração entre estudantes e docentes de diversas áreas de atuação, com a participação de vários profissionais técnicos da universidade. A ação desenvolvida evidenciou também que um grupo de pesquisa, além de pesquisar e realizar reuniões, pode e deve implementar ações práticas com a população envolvendo a comunidade com questões importantes para a saúde. É uma forma de instigar a cidadania e a participação popular.

\section{CONSIDERAÇÕES FINAIS}

A realização da atividade permitiu envolver estudantes de diversos cursos, docentes e técnicos da universidade, oportunizando a reflexão sobre o conceito de saúde e a auto avaliação de saúde desses participantes. Os conceitos compartilhados e discutidos sobre saúde aproximamse tanto do proposto pela OMS, como também das proposições da $8^{\mathrm{a}} \mathrm{CNS}$ que valoriza os determinantes da saúde, vinculando saúde com a situação de vida de cada indivíduo e dependente de fatores como direito e acesso a alimentação, moradia, emprego, lazer, sistema de saúde, entre outros.

Quando questionados sobre como avaliavam sua própria saúde, alguns participantes a consideraram boa, outros regular, e houve aqueles que afirmaram estar ruim. Entre os fatores que influenciam a saúde e QV estão a alimentação saudável, a prática de exercícios físicos, a saúde mental, o estresse e a falta de tempo devido à rotina acadêmica, a mudança de cidade e a saída da casa dos pais. Torna-se imprescindível a continuidade de atividades como essa que oportunizem discussões sobre diversos temas, tanto na universidade como em outros espaços comunitários.

\section{REFERÊNCIAS}

ALMEIDA, N.D. A saúde no Brasil, impasses e desafios enfrentados pelo Sistema Único de Saúde - SUS. Revista Psicologia e Saúde, v.5, n.1, p.01-09, jan./jun. 2013. Disponível em: http://pepsic.bvsalud.org/pdf/rpsaude/v5n1/v5n1a02.pdf. Acesso em: 16 dez. 2018.

ARAÚJO, J.S.; XAVIER, M. P. O conceito de saúde e os modelos de assistência: considerações e perspectivas em mudança. Revista Saúde em Foco, Teresina, v.1, n.1, art. 10, p.137- 49, jan. / 
Conceitos e práticas em saúde: a enfermagem comemorando o Dia Internacional da Saúde

jul. 2014. Disponível em:

http://www4.fsanet.com.br/revista/index.php/saudeemfoco/article/viewFile/326/382\&gws_rd =cr\&ei=ycB-WJeGNMebwASnrrrwAg. Acesso em: 16 dez. 2018.

BEZERRA, I.M.P.; SORPRESO, I.C.E. Conceitos de saúde e movimentos de promoção da saúde em busca da reorientação de práticas. J. Hum. Growth Dev. v.26, n.1, p.11-20, 2016, Disponível em: http:/ / pepsic.bvsalud.org/scielo.php?script=sci_arttext\&pid=S0104$12822016000100002 \& \operatorname{lng}=$ pt\&nrm=iso. Acesso em 06 jun. 2018.

BOORSE, C. Health as a theoretical concept. Philosophy of Science, v.44, p.542-73, 1977. Disponível em: https://bioetyka.uw.edu.pl/wpcontent/uploads/2014/10/06_BoorseHealthConcept.pdf. Acesso em: 17 dez. 2018.

BRASIL. $8^{a}$ Conferência Nacional de Saúde. Relatório Final. 1986. Disponível em: http://bvsms.saude.gov.br/bvs/publicacoes/8_conferencia_nacional_saude_relatorio_final.pdf. Acesso em: 03 jun. 2018.

BRASIL. Constituição da República Federativa do Brasil, 1988. Disponível em: http://www.planalto.gov.br/ccivil_03/Constituicao/Constituicao.htm. Acesso em: 28 maio 2018.

BUSS, P.M.; PELLEGRINI FILHO, A. A Saúde e seus Determinantes Sociais. Physis [online]. Rio de Janeiro, v.17, n.1, p.77-93, 2007. Disponível em: http://www.scielo.br/pdf/physis/v17n1/v17n1a06.pdf. Acesso em: 03 jun. 2018.

DELIENS, T. et al. Determinants of physical activity and sedentary behaviour in university students: a qualitative study using focus group discussions. BMC Public Health, v.15, n.201, p.1-9, 2015. Disponível em:

https://bmcpublichealth.biomedcentral.com/track/pdf/10.1186/s12889-015-1553-4. Acesso em: 30 jul. 2019.

FLECK, M.P.A. O instrumento de avaliação de qualidade de vida da Organização Mundial da Saúde (WHOQOL-100): características e perspectivas. Ciênc. saúde coletiva. Rio de Janeiro, v.5, n.1, p.33-8, 2000. Disponível em: http://www.scielo.br/scielo.php?script=sci_arttext\&pid=S1413$81232000000100004 \& \operatorname{lng}=$ en\&nrm=iso. Acesso em: $16 \mathrm{dez} .2018$.

FONTES, A.C.D.; VIANNA R.P.T. Prevalência e fatores associados ao baixo nível de atividade física entre estudantes universitários de uma universidade pública da região Nordeste - Brasil.

Rev. bras. epidemiol. São Paulo, v.12, n.1, p. 20-29, mar. 2009. Disponível em: http://www.scielo.br/scielo.php?script=sci_arttext\&pid=S1415790X2009000100003\&lng=en\&nrm=iso. Acesso em: 28 maio 2018.

GRANER, K.M.; CERQUEIRA, A.T.A.R. Revisão integrativa: sofrimento psíquico em estudantes universitários e fatores associados. Ciênc. saúde coletiva. v.24, n.4, p.1327-46, 2019. Disponível em: http:/ /www.scielo.br/scielo.php?script=sci_abstract\&pid=S1413$81232019000401327 \& \operatorname{lng}=$ en\&nrm=iso\&tlng=pt. Acesso em: 30 jul. 2019.

LANSINI, L.C. et al. Nível de sedentarismo entre estudantes universitários do Rio Grande do Sul e os possíveis fatores associados. O Mundo da Saúde. v.41, n.3, p.267-74, 2017. Disponível em: http://www.revistamundodasaude.com.br/uploads/20160114.PDF Acesso em: 30 jul. 2019. 
Conceitos e práticas em saúde: a enfermagem comemorando o Dia Internacional da Saúde

MOURA, A.F.; LIMA, M.G. A reinvenção da roda: roda de conversa: um instrumento metodológico possível. Rev. temas educ. João Pessoa, v.23, n.1, p.98-06, jan./jun. 2014. Disponível em: http://www.periodicos.ufpb.br/index.php/rteo/article/view/18338. Acesso em: 13 maio 2018.

NAHAS, M.V. Estilo de vida e o conceito de saúde positiva. Fundamentos da aptidão física relacionada à saúde. Florianópolis: Editora da UFSC, 1989.

ORGANIZAÇÃO MUNDIAL DA SAÚDE. Constituição da Organização Mundial da Saúde, 1946. Disponível em: http://www.direitoshumanos.usp.br/index.php/OMSOrganiza $\% \mathrm{C} 3 \% \mathrm{~A} 7 \% \mathrm{C} 3 \% \mathrm{~A} 3 \mathrm{o}-\mathrm{Mundial}-\mathrm{da}-\mathrm{Sa} \% \mathrm{C} 3 \% \mathrm{BAde} /$ constituicao-da-organizacao-mundialda-saude-omswho.html. Acesso em: 25 maio 2018.

PADOVANI, R.C. et al. Vulnerabilidade e bem-estar psicológicos do estudante universitário. Rev. bras. ter. cogn. [online]. v.10, n.1, p.2-10, 2014. Disponível em: http://pepsic.bvsalud.org/scielo.php?script $=$ sci_abstract\&pid=S180856872014000100002\&lng=pt\&nrm=iso. Acesso em: 30 jul. 2019.

RAMOS, A.P.Q.R. et al. Prevalência e fatores associados à prática de atividade física entre universitários da saúde. Rev. Aten. Saúde. v.17, n.59, p.10-18, jan./mar., 2019. Disponível em: https://seer.uscs.edu.br/index.php/revista_ciencias_saude/article/view/5728. Acesso em: 30 jul. 2019.

SILVA, E.C.; HELENO, M.G.V. Qualidade de Vida e Bem-Estar Subjetivo de Estudantes Universitários. Revista Psicologia e Saúde, v.4, n.1, p.69-76, jan. 2012. Disponível em: http://www.gpec.ucdb.br/pssa/index.php/pssa/article/view/126. Acesso em: 28 maio 2018.

SCLIAR, M. História do Conceito de Saúde. Physis [online], Rio de Janeiro, v.17, n.1, p.29-41, mar. 2007. Disponível em: http://www.scielo.br/pdf/physis/v17n1/v17n1a03.pdf. Acesso em: 12 maio 2018.

VICTOR. Dia Mundial da Saúde: o que é, como surgiu, objetivos, tema de 2018. 2018. Disponível em: https://minutosaudavel.com.br/dia-mundial-da-saude/ . Acesso em: 25 maio 2018.

WORLD HEALTH ORGANIZATION. The Ottawa Charter for Health Promotion. Geneva: World Health Organization; 1986. Disponível em: https://www.who.int/healthpromotion/conferences/previous/ottawa/en/. Acesso em: 28 maio 2018.

WORLD HEALTH ORGANIZATION. Quality of life assessment an annotated bibliography. Geneva: World Health Organization; 1994. Disponível em: https://apps.who.int/iris/bitstream/handle/10665/61629/WHO_MNH_PSF_94.1.pdf?sequenc e=1\&isAllowed $=$ y. Acesso em: 02 ago. 2019.

Recebido em: 17/12/2018

Aceito em: 05/08/2019 